\title{
Spatiotemporal Modeling of COVID-19 Spread in Built Environments
}

\author{
Paula Gomez \\ Georgia Tech Research Institute | USA | Paula.Gomez@gtri.gatech.edu \\ Khatereh Hadi \\ Georgia Tech Research Institute | USA | Khatereh.Hadi@gtri.gatech.edu \\ Olga Kemenova \\ Georgia Tech Research Institute | USA | Olga.Kemenova@gtri.gatech.edu \\ Matthew Swarts \\ Georgia Tech Research Institute | USA | Matthew.Swarts@gtri.gatech.edu
}

\begin{abstract}
This research proposes a Spatiotemporal Modeling approach to understand the role of architecture, specifically the built environment, in the COVID-19 pandemic. The model integrates spatial and temporal parameters to calculate the probability of spread of and exposure to SARS-CoV-2 virus (responsible of COVID-19 disease) due to the combination of four aspects: Spatial configuration, organizational schedules, people's behavior, and virus characteristics. Spatiotemporal Modeling builds upon the current models of building analytics for architecture combined with predictive models of COVID-19 spread. While most of the current research on COVID-19 spread focuses on mathematical models at regional scales and the CDC guidelines emphasizing on human behavior, our research focuses on the role of buildings in this pandemic, as the intermediate mechanism where human and social activities occur. The goal is to understand the most significant parameters that influence the virus spread within built environments, including human-to-human, fomite (surface-to-human), and airborne ways of transmission, with the purpose of providing a comprehensive parametric model that may help identify the most influential design and organizational decisions for controlling the pandemic. The proof-of-concept study is a healthcare facility.
\end{abstract}

Keywords: Spatiotemporal modeling; Agent-based simulation; COVID-19; Virus spread; Built environments; Human behavior; Social distancing.

\section{INTRODUCTION}

Since the beginning of the COVID-19 pandemic, several studies have modeled the virus spread dynamics with the purpose of forecasting the number of COVID-19 infections, identifying the most significant factors to control them. These virology-centric models utilize various predictive mathematical, statistical and agent-based models to project the spread of the virus, under certain conditions. These predictive models include different categories of clinical, social and environmental factors to characterize the outbreak. Most of the current modeling efforts focus on understanding the virus spread patterns at large scales such as urban or regional scales, however they do not incorporate the building scale, where most of the infections actually occur.

To characterize the epidemic of COVID-19, most of the existing models use generalized Susceptible-ExposedInfectious-Removed' (SEIR) models by defining different states for susceptible, exposed, infectious and removed populations (Biswas \& Sen, 2020; Hamzah et al., 2020; Huang et al., 2020; Jia et al., 2020; Kim et al., 2018; Lin et al., 2020; Peng et al., 2020; Rabajante, 2020). These models usually assume an initial total population and an initial susceptible population, along with recruitment, transmission, incubation, recovery and mortality rates. In some cases, the SEIR models have been further expanded to include other factors in the model that can affect the spread including exposure time, level of Personal Protective Equipment (PPE), including mask wearing and handwashing (Rabajante, 2020), average close contacts, quarantined time, early quarantine, timely disinfection (Peng et al., 2020), severe condition rate, emigration rate (Lin et al., 2020), and vaccination (Jia et al., 2020). A few of the existing predictive models also explore the impacts of controlling policies and restricting measures such as efficiency of quarantine and isolation (Nadim et al., 2020), government action strength (e.g. Holiday extension, travel restriction), intensity of responses to policies (Lin et al., 2020), mobility control (Ma, 2020), city closure strategies (Huang et al., 2020), restriction on international travels, and school closure (Chang et al., 2020).

Although the built environment may play a significant role in the virus spread, the inclusion of spatial factors in COVID-19 modeling studies has been limited to a few measures including social distancing (Giuliani et al., 2020; Kim et al., 2018), distance from the epicenters (Rabajante, 2020), and geographical location (Giuliani et al., 2020; Ma, 2020). The most current research shows how different 
attributes of the built environment, such as virus stability on surfaces, temperature and humidity (Jia et al., 2020), and attributes of behavior, such as occupancy rates and PPE levels (Jones, N. R., Qureshi, Z. U., Temple, R. J., Larwood, J. P., Greenhalgh, T., \& Bourouiba, L., 2020). are related to the probabilities of virus transmission. In this context, our research proposes to build a model that integrates: 1) Virus data from experimental research; 2) Human behavior, which is the key mechanism for virus transmission; and 3) The building, which offers the conditions for the virus transmission to occur.

The building layout and therefore its program, schedule and occupancy rates, characterize the building analytics. These include the most probable occupied spaces and areas, the most probable circulations routes, and occupancy rates, define the potential use of the space. Dynamic environmental conditions such as temperature, humidity and lighting conditions, also have an impact on this specific spatiotemporal model for virus spread.

Human behavior plays a significant role in the pandemic. A sick person will spread the virus depending on several factors, including the severity of their symptoms, the activities and actions performed, and the use of PPE. First, the distribution of sick population is approximately $30 \%$ asymptomatic and $55 \%$ with mild symptoms. The remaining $15 \%$ require hospitalization. The duration of a contagion period increases from 14 to 25 days based on the severity of the symptoms (Lauer, S. A., Grantz, K. H., Bi, Q., Jones, F. K., Zheng, Q., Meredith, H. R.,Azman N., Reigh, \& Lessler, J., 2020). Second, the distance to the infection source is another determining factor in the spread of coronavirus. Although a 6-feet distance was initially suggested for social distancing measures, the current evidence shows that it depends on the exhalation action being performed by the agent-source. If the agent singing, or screaming, the distance the virus may travel is significantly longer (in the case of no PPE) compared to silent or talking agents (Jones et al., 2020).

COVID-19 recent research presents ample evidence on the impact of different surface materials on SARS-CoV-2 stability and persistence. SARS-CoV-2 virus, responsible of COVID-19 disease, has a longer stability on surface materials such as plastic, stainless steel, metal and wood compared to copper (Chin et al., 2020; Dietz et al., 2020; Kampf et al., 2020; van Doremalen et al., 2020).

Combining the effect of surface materials with environmental conditions, such as temperature, relative humidity levels, and light conditions presents a variation on SARS-CoV-2 stability on surfaces in a particular environment. For all materials, the virus stability is longer in lower temperatures (Casanova et al., 2010; Chin et al., 2020; Pawar et al., 2020). Survival rates (half-lives) of SARS-CoV-2 at different temperatures range between 1.7 and 2.7 days at $20^{\circ} \mathrm{C}$, reducing to less than 24 hours when temperature is elevated to $40^{\circ} \mathrm{C}$ on some surfaces. (Riddell, S., Goldie, S., Hill, A. et al., 2020). The virus stability is also longer at low (10\%-40\%) and high (60\%$100 \%$ ) relative humidity levels, and it is shorter at moderate relative humidity levels (40\%-60\%) (Dietz et al., 2020; Moriyama et al., 2020). Exposure to light and direct sunlight also decreases the coronavirus stability time (Schuit et al., 2020). Stability could reach longer survival times, even weeks, in dark spaces (Riddell, S., Goldie, S., Hill, A. et al.,
2020). For example, common surfaces such as glass, stainless steel, paper and polymer banknotes at $20^{\circ} \mathrm{C}$, in dark conditions, allow virus stability for up to 28 days (Riddell et al., 2020). *Please, note that these studies were ran in laboratory conditions.

Also, the airborne diffusion of infected droplets can spread beyond the 6-feet distance (Setti et al., 2020). The coronavirus droplets can be transmitted through aerosols up to 23-27 feet at peak exhalation speeds (Bourouiba, 2020; Guo et al., 2020). The virus survival time in the air is approximately 3 hours (van Doremalen et al., 2020), and the direction of air flow and airflow zoning also impacts the viral spread (CDC, 2020; Liu et al., 2020; Lu et al., 2020).

The above-mentioned studies emphasize the impact of building and environmental parameters on the virus spread. To this date, the existing SARS-CoV-2 spread modeling studies have not included such parameters in the simulation of the spread. Our study addresses this gap by creating and implementing a comprehensive spatiotemporal model which includes these viral characteristics, spatial parameters, as well as organizational schedules and their building occupancy patterns to understand the virus spread. The end goal is to provide design and organizational guidelines to help control the virus spread for reopening spaces.

\section{METHODOLOGY}

Spatiotemporal modeling integrates several modeling and simulation methods, combining spatial analytics with behavioral and building analytics, integrating operational parameters, and environmental characteristics. This research extends the spatiotemporal occupancy in building settings research by Gomez-Zamora (2017, 2019) For COVID-19 spread specification of spatiotemporal modeling, we included materials specifications and virus characteristics. This approach, however, can be applied to other analytics that requires time as a variable for spatial analytics.

The spatiotemporal model superimposes four layers of information:

a. Spatial analytics (Figure 1)

b. Operational analytics (Figure 2)

c. Agent-based simulation (Figure 3)

d. Virus characteristics and preventive policies

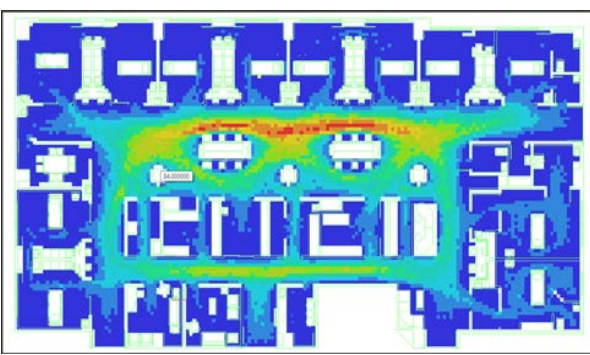

Figure 1. Most probable occupied spaces in red (Sample ICU layout: from Award Winning ICU Designs 1992-2012, Society of Critical Care Medicine)

In order to better understand the dynamics of COVID-19 spread within the built environments, we identified a set of parameters that have been proven to have an impact on the virus spread. The weight of each parameters is yet to 
be determined. These parameters are categorized as Virus parameters, Behavior Parameters, and Building parameters. Building parameters include surface materials characteristics, exposure to direct sunlight layouts, most probable occupied areas, most probable circulations, ambient temperature and relative humidity level, as well as airflows dynamics. Behavior parameters include occupancy level, distance between users, schedule, time of occupancy, sequence of places visited, activity performed, and PPE level. Virus parameters include survival time on different materials, including air, by temperature, light and humidity, number of cases by total population in the area of the building, distance that the virus can travel due to particle size, and based on a specific action: silence, talking, sneezing, coughing, and screaming or signing (Jones et al, 2020).

\section{MODELING PARAMETERS}

The modeling strategy focused on implementing the parameters that play significant roles in the virus spread dynamic on a comprehensive multi-dimensional modeling approach that included the following parameters:

\section{Building Parameters:}
a. Layout
b. Probability of occupancy
c. Probability of surface touch
d. Materials of surfaces (link to virus survival time)
e. Direct sunlight exposure (ranges)
f. Temperature and humidity levels (ranges) Agents:
a. Agent role (Doctor, Nurse, Patient, Visitor)
b. Health status (Healthy or Sick (Spreader))
c. Sick agent: asymptomatic, mild symptoms, severe symptoms, or critical symptoms. The level of symptoms is linked to the period of time that the agent would act as a spreader ( 14 - 25 days)
d. PPE levels (No PEE, Level 1-5)
e. Distance from/to source $(0-6$ feet $)$
f. Time of exposure (exponentially increasing risk after 15 min within 24 hours)

Virus Characteristics:

a. Virus travel distance from the source, depending on particle size.

b. Time that a particle size floats in the air.

c. Survival time on materials and air (12-72 hours)

d. Survival time under certain temperature, humidity, and lighting conditions (12hrs.-28 days).

e. Number of known COVID-19 cases by population, in the area of the building.

f. Asymptomatic/Symptomatic population distribution.

After identifying spatial, behavior and virus parameters from the review of current literature, we created an agentbased simulation model that incorporates the above behavior parameters and ongoing workflows within the environment, in addition to virus characteristics, in different scenarios for risk comparison.

\section{AGENT-BASED SIMULATION}

We implemented a proof of concept of the spatiotemporal model using an intensive care unit (ICU). The inputs of our model included the ICU layout, spatial parameters, and patient care processes and staff workflows within the ICU representing the building operations. The care processes and workflows included standard patient care activities such as patient assessment, medication administration, nutrition delivery, and procedures. A set of agents were created to represent care team members including 6 bedside nurses (responsible for direct patient care), a nurse manager (managing the unit), 2 doctors, 2 respiratory specialists and a receptionist. The agents were set to move between different rooms and areas (patient rooms, central nurse stations, distributed nurse stations, clean utility rooms, medication room, nutrition room, storage, soiled utility, etc.) to perform their daily tasks.

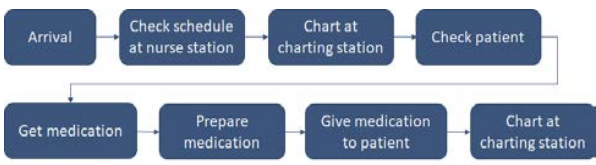

Figure 2. Sample of sequence of activities and schedules of the healthcare operations

We simulated staff movements in the spaces (Figures 3 and 4) and their interactions with the built environment such as touching various surfaces while working, and occupying spaces with different characteristics, associated with virus spread probability. The simulation model was created in a parametric environment (Grasshopper). For simulating the agents' movements, we used PedSim Pro add-on (https://www.pedsim.net).

For each agent, we defined a role and a set of tasks and destinations, as well as a set of attributes including the health status and PPE level. From the simulation, we extracted the agents' positions in time $(x, y, t)$.

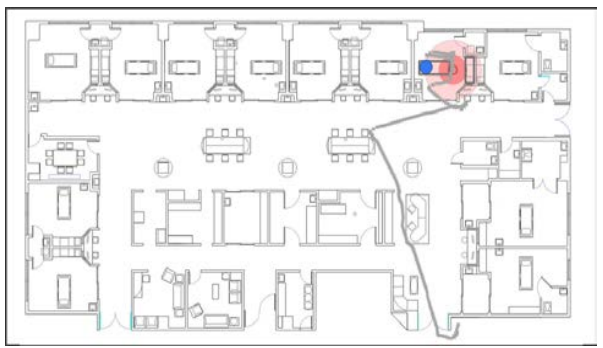

Figure 3. Agent's movement path is shown with a grey line. The blue dot represents a healthy agent and the red dot a sick agent (radius of influence of 6-ft).

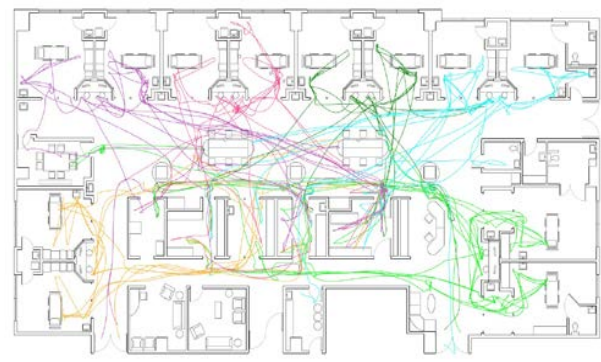

Figure 4. Paths generated by movement of agents in the overall simulation. 


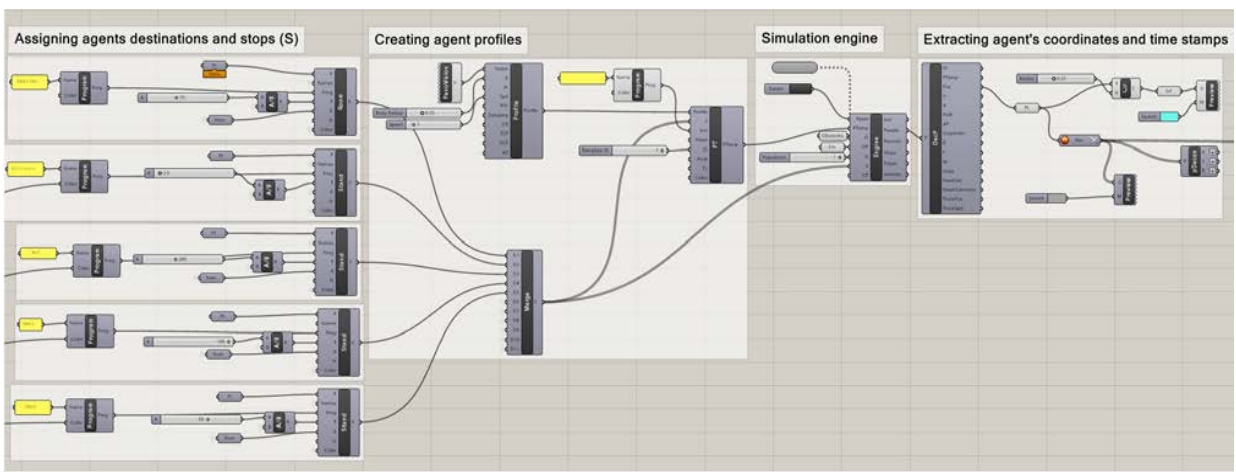

Figure 5. Agent's destinations and stops, Agent profiles; Simulation engine and Agents' location extraction components in Grasshopper.

The agent's health status is healthy or sick. A sick agent is defined as a virus spreader during a period of time. Spreaders could be symptomatic or asymptomatic, and the distribution of sickness levels over the total populations is determined by current research, as well as the viral load, per agent, over time, which is also determined by a specific statistical distribution (Kilpatrick, A.M., 2020).

A healthy agent is defined as (virus) collector. The probability of exposure of this agent depends on several factors, such as the time of exposure to virus sources (spreaders or contaminated air), the distance to the source, and probability of fomite contagion (surface-touch followed by face-touch). According to CDC Public Health guidelines for contact tracing, the probability of contagion risk for close contacts increase over time after 15 minutes of accumulated exposure within 24 hours (Appendices, cdc.gov). The aforementioned parameters are affected by ambient conditions, such as temperature, humidity levels, sunlight, as well as protective protocols such as use of PPE, frequent handwashing, air filtration and surface cleaning.

The simulation model integrates spatial and temporal parameters into a comprehensive model in Grasshopper (Figures 5), to generate two metrics as outputs of the model:

a. The Probability of Spatial Viral Contamination is an attribute of space, assigned to a spatial cell (1 ft3), at a specific time.

b. The Probability of Agent Exposure is an attribute of the agent, assigned to an agent at a specific location in time, calculated based on the sequence of activities and actions, and the spatial viral contamination.

We plan on testing several scenarios to compare the probabilities of virus contamination and agent exposure in buildings areas, as agents move through the space.

\section{PRELIMINARY RESULTS}

As this is a spatiotemporal modeling, the probability of virus contamination on air and surfaces varies over time, depending on the combination of parameters described in the previous section. The probability of exposure of an agent is directly related to the sequence of activities and exposure to the contaminated spaces or sources, over a period of time.
The probability of occupancy is modeled utilizing data from two sources, the spatial analyses of the layout and operational analytics. The probability of fomite contamination is calculated based on the probability of an infected agent touching a surface and data on virus survival time by material, at specific environmental conditions. The probabilities of a healthy agent's risk of exposure is calculated based on the utilized PPE level, interaction with infection source (fomite or spreader), source contamination levels, actions performed, exposure time to the source. Synthetic parameters are generated based on studies conducted for other viruses where the data on SARS-CoV2 was unavailable. Exposure times necessary to inhale a critical number of virus particles are calculated based on the study conducted for seasonal cases of influenza (Yan et al., 2018).

In Table 1, the probability of agent risk of exposure due to a specific contaminated surface, during a period of time, is presented as an example. First, the probability of spatial viral contamination is calculated based on the time a spreader previously spent at that location, considering the probability of virus spread based on her health condition, PPE level, and action performed. The material type, ambient temperature and humidity (constant for this proofof-concept study), and frequency of cleaning, will be factors for the virus stability on that surface. Second, the calculation of probability of risk of a healthy agent spending time at the same location, includes the PPE level, action performed, time of exposure, and probability of face-touch. The final probability of risk for that agent is the accumulative probability over time, within 24 hours.

For this current spatiotemporal model, we defined five levels of PPE: 0: No PPE; 1: cloth mask; 2 : surgical mask; 3: N95 mask; 4: N95+shielding+gloves; Level 5: L4+cover These levels and probabilities of spreading and contracting (the virus) are defined by current research.

The final exposure score for the agent is calculated based on the accumulated time of exposure and the probability of exposure. The agent change status to sick when it scores 100 (*this is a scale defined for this simulation only, and it does not directly apply to reality). Sensitivity analysis is not implemented in the current model. The concept of viral dosing also needs to be specified in the next research stage. 
Table 1. Sample of a Probability of Agent Exposure as it moves through space, at each time step (x, y, t).

\begin{tabular}{|c|c|c|c|c|c|c|c|c|c|c|c|c|c|c|c|}
\hline 으 & $\frac{0}{0}$ & $\begin{array}{l}\frac{n}{3} \\
\frac{1}{\pi} \\
\text { 01 } \\
\frac{5}{10} \\
\frac{1}{10} \\
\frac{1}{1}\end{array}$ & 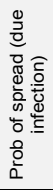 & 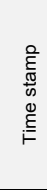 & $\times$ & $>$ & 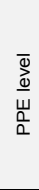 & 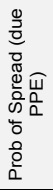 & 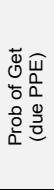 & 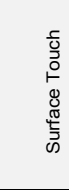 & 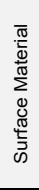 & 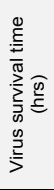 & 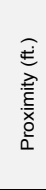 & 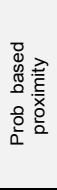 & 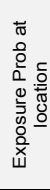 \\
\hline${ }_{7}{ }^{2} \mid$ & Nurse & Healthy & 0 & 0 & 16.5 & -1.1 & 3 & 0.25 & 0.75 & FALSE & 0 & 3 & $>6$ & 0 & 0 \\
\hline 7 & Nurse & Healthy & 0 & 1 & 16.5 & -1.4 & 3 & 0.25 & 0.75 & FALSE & 0 & 3 & $>6$ & 0 & 0 \\
\hline 7 & Nurse & Healthy & 0 & 2 & 16.5 & -0.9 & 3 & 0.25 & 0.75 & FALSE & 0 & 3 & $>6$ & 0 & 0 \\
\hline 7 & Nurse & Healthy & 0 & 3 & 16.4 & -0.8 & 3 & 0.25 & 0.75 & FALSE & 0 & 3 & $>6$ & 0 & 0 \\
\hline 7 & Nurse & Healthy & 0 & 4 & 16.4 & -0.7 & 3 & 0.25 & 0.75 & FALSE & 0 & 3 & $>6$ & 0 & 0 \\
\hline 7 & Nurse & Healthy & 0 & 5 & 16.4 & -0.5 & 3 & 0.25 & 0.75 & FALSE & 0 & 3 & $>6$ & 0 & 0 \\
\hline 7 & Nurse & Healthy & 0 & 6 & 16.4 & -0.3 & 3 & 0.25 & 0.75 & FALSE & 0 & 3 & 3 & 0.25 & 0.18 \\
\hline 7 & Nurse & Healthy & 0 & 7 & 16.4 & -0.1 & 3 & 0.25 & 0.75 & TRUE & 4 & 72 & 3 & 0.25 & 0.18 \\
\hline 7 & Nurse & Healthy & 0 & 8 & 16.4 & 0 & 3 & 0.25 & 0.75 & TRUE & 4 & 72 & 0 & 0.75 & 0.56 \\
\hline 7 & Nurse & Healthy & 0 & 9 & 16.4 & 0.2 & 3 & 0.25 & 0.75 & TRUE & 4 & 72 & 0 & 0.75 & 0.56 \\
\hline 7 & Nurse & Healthy & 0 & 10 & 16.4 & 0.4 & 3 & 0.25 & 0.75 & TRUE & 4 & 72 & 0 & 0.75 & 0.56 \\
\hline
\end{tabular}

\section{DISCUSSION}

This artcile presents a comprehensive spatiotemporal modeling approach for understangind the dynamics in a built environment, comprising building analytics and organizational analytics, including human behavior. This particular application extends the spatiotemporal modeling research towards COVID-19 spread, through space, surfaces and agents. This specific model is loaded with virus characteristics, with the purpose of creating a parametric model for understanding the sensitivity of the built environment and human behavior variables on the COVID-19 spread. The end-goal is to help define spatial and organizational guidelines for safe re-opening of spaces. Even though this research is specifically in Healthcare, the implementation approach can be extended to any architectural programs.

The outcomes of this research include the probability of COVID-19 spread and agents' exposure, based on a set of given paramters (listed in the Modeling Parameters section) for a certain layout. The proposed spatiotemporal model provides a framework to evaluate various design and operational scenarios, with the purpose of understanding which strategies would more effectively help reduce virus exposure by providing design changes or preventative protocols (i.e. cleaning frequency, new physical barriers, and circulation direction).

As none of the existing software allows the integration of such expert knowledge, we designed the spatiotemporal modeling approach based on spatial modeling integrated with agent-based models. We customized the existing platforms to integrate both, spatial and agent modeling, with environmental attributes and virus characterization models, described in the previous sections. The current spatiotemporal modeling of COVID-19 spread includes most of the building and environmental factors. These factors will probablly update as most current research on SARS-CoV-2 becomes avaiblale. Therefore, the proposed framwork is parametric, allowing the integration of additional and updated parameters, changing the threshoulds and the sensitivity of the parameters as the COVID-19 research refines and expands. One challenge we forsee is the validation of this approach with real data. For overcoming this challenge, we propose that the owners and users can parametrcially define the design and organizational modifications they would like to implement, to analyze possible scenarios, and compare the risk.

Future work includes another modeling layer: airflow dynamics, implemented using a Computational Fluid Dynamics (CFD) approach. The purpose is to include the most recent, yet controversial, evidence on aerosols spread, as it has been recently identified as one of the dominand route for the transmission of SARS-CoV-2 (Zhang, R., Li, Y., Zhang, A. L., Wang, Y., and Molina, M. J., 2020). Future research also includes the analysis of two types of HVAC systems, and their impact on airflow dynamics, as it modifies our calculations on virus spread probabilities. This analytics will offer a third level of solutions for controlling the spread: Technical solutions.

\section{ACKNOWLEDGEMENTS}

This research was funded by the Internal Research and Development (IRAD) ATAS Program, at the Georgia Tech Research Institute. We thank the program directors and internal advisors for their support and valuable feedback

\section{REFERENCES}

Biswas, K., \& Sen, P. (2020). Space-time dependence of corona virus (COVID-19) outbreak. http://arxiv.org/abs/2003.03149

Bourouiba, L. (2020). Turbulent Gas Clouds and Respiratory Pathogen Emissions: Potential Implications for Reducing Transmission of COVID-19. JAMA. https://doi.org/10.1001/jama.2020.4756

Casanova, L. M., Jeon, S., Rutala, W. A., Weber, D. J., \& Sobsey, M. D. (2010). Effects of air temperature and relative humidity on coronavirus survival on surfaces. Applied and Environmental Microbiology, 76(9), 2712-2717. https://doi.org/10.1128/AEM.02291-09

CDC. (2020). Interim Infection Prevention and Control Recommendations for Healthcare Personnel During the Coronavirus Disease 2019 (COVID-19) Pandemic.

Chang, S. L., Harding, N., Zachreson, C., Cliff, O. M., \& Prokopenko, M. (2020). Modelling transmission and control of the COVID-19 pandemic in Australia. http://arxiv.org/abs/2003.10218

Chin, A. W. H., Chu, J. T. S., Perera, M. R. A., Hui, K. P. Y., Yen, H.-L., Chan, M. C. W., Peiris, M., \& Poon, L. L. M. (2020). Stability of SARS-CoV-2 in different environmental conditions. The Lancet Microbe, $0(0)$. https://doi.org/10.1016/S2666-5247(20)30003-3 
Dietz, L., Horve, P. F., Coil, D. A., Fretz, M., Eisen, J. A., \& Van Den Wymelenberg, K. (2020). 2019 Novel Coronavirus (COVID-19) Pandemic: Built Environment Considerations To Reduce Transmission. MSystems, 5(2). https://doi.org/10.1128/mSystems.00245-20

Giuliani, D., Dickson, M. M., Espa, G., \& Santi, F. (2020). Modelling and predicting the spatio-temporal spread of Coronavirus disease 2019 (COVID-19) in Italy. http://arxiv.org/abs/2003.06664

Gomez Zamora, P. A. (2017). Spatiotemporal occupancy in building settings (Doctoral dissertation, Georgia Institute of Technology).

Gomez-Zamora, P., Bafna, S., Zimring, C., Do, E., \& Romero Vega, M. (2019). Spatiotemporal Occupancy for Building Analytics.

Guo, Z.-D., Wang, Z.-Y., Zhang, S.-F., Li, X., Li, L., Li, C., Cui, Y., Fu, R.-B., Dong, Y.-Z., Chi, X.-Y., Zhang, M.-Y., Liu, K., Cao, C., Liu, B., Zhang, K., Gao, Y.-W., Lu, B., \& Chen, W. (2020). Aerosol and Surface Distribution of Severe Acute Respiratory Syndrome Coronavirus 2 in Hospital Wards, Wuhan, China, 2020. Emerging Infectious Diseases, 26(7). https://doi.org/10.3201/eid2607.200885

Hadi, K. (2020) Agent-based Simulation For Evaluating The Impacts Of Design On Nurses' Spatiotemporal Experience. (Doctoral dissertation, Georgia Institute of Technology).

Hamzah, F. A. B., Lau, C. H., Nazri, H., Ligot, D. V., Lee, G., Tan, C. L., Shaib, M. K., Zaidon, U. H. B., Abdullah, A. B., Chung, M. H., Ong, C. H., Chew, P. Y., \& Salunga, R. E. (2020). CoronaTracker: World-wide COVID-19 Outbreak Data Analysis and Prediction. The Bulletin of the World Health Organization, March 18th

Huang, Y., Yang, L., Dai, H., Tian, F., \& Chen, K. (2020). Bull World Health Organ. E-pub: 16. https://doi.org/10.2471/BLT.20.251561

Jia, J., Ding, J., Liu, S., Liao, G., Li, J., Duan, B., Wang, G., \& Zhang, R. (2020). Modeling the Control of COVID-19: Impact of Policy Interventions and Meteorological Factors. http://arxiv.org/abs/2003.02985

Kampf, G., Todt, D., Pfaender, S., \& Steinmann, E. (2020). Persistence of coronaviruses on inanimate surfaces and their inactivation with biocidal agents. In Journal of Hospital Infection (Vol. 104, Issue 3, pp. 246-251). W.B. Saunders Ltd. https://doi.org/10.1016/j.jhin.2020.01.022

Kim, Y., Ryu, H., \& Lee, S. (2018). Agent-based modeling for super-spreading events: A case study of mers-cov transmission dynamics in the republic of korea. International Journal of Environmental Research and Public Health, 15(11). https://doi.org/10.3390/ijerph15112369

Lauer, S. A., Grantz, K. H., Bi, Q., Jones, F. K., Zheng, Q., Meredith, H. R., ... \& Lessler, J. (2020). The incubation period of coronavirus disease 2019 (COVID-19) from publicly reported confirmed cases: estimation and application. Annals of internal medicine, 172(9), 577-582.

Lin, Q., Zhao, S., Gao, D., Lou, Y., Yang, S., Musa, S. S., Wang, M. H., Cai, Y., Wang, W., Yang, L., \& He, D. (2020). A conceptual model for the coronavirus disease 2019 (COVID-19) outbreak in Wuhan, China with individual reaction and governmental action. International Journal of Infectious Diseases, 93, 211-216. https://doi.org/10.1016/j.ijid.2020.02.058

Liu, Y., Ning, Z., Chen, Y., Guo, M., Liu, Y., Gali, N. K., Sun, L., Duan, Y., Cai, J., Westerdahl, D., Liu, X., Xu, K., Ho, K., Kan, H., Fu, Q., \& Lan, K. (2020). Aerodynamic analysis of SARS-CoV-2 in two Wuhan hospitals. Nature. https://doi.org/10.1038/s41586-020-2271-3

Lu, J., Gu, J., Li, K., Xu, C., Su, W., Lai, Z., Zhou, D., Yu, C., Xu,
B., \& Yang, Z. (2020). COVID-19 Outbreak Associated with Air Conditioning in Restaurant, Guangzhou, China, 2020. Emerging Infectious Diseases, 26(7). https://doi.org/10.3201/eid2607.200764

Ma, Z. (2020). Spatiotemporal fluctuation scaling law and metapopulation modeling of the novel coronavirus (COVID19) and SARS outbreaks. http://arxiv.org/abs/2003.03714

Marr, Miller, Prather, Haas, Bahnfleth,Corsi, Tang, Hermann, Pollitt et al. (2020) https://tinyurl.com/FAQ-aerosols

Moriyama, M., Hugentobler, W. J., \& Iwasaki, A. (2020). Seasonality of Respiratory Viral Infections. Annual Review of Virology, 7(1). https://doi.org/10.1146/annurev-virology012420-022445

Nadim, S. S., Ghosh, I., \& Chattopadhyay, J. (2020). Short-term predictions and prevention strategies for COVID-2019: A model based study. http://arxiv.org/abs/2003.08150

Pawar, S., Stanam, A., Chaudhari, M., \& Rayudu, D. (2020). Effects of temperature on COVID-19 transmission. MedRxiv, 2020.03.29.20044461 https://doi.org/10.1101/2020.03.29.20044461

Peng, L., Yang, W., Zhang, D., Zhuge, C., \& Hong, L. (2020) Epidemic analysis of COVID-19 in China by dynamical modeling. http://arxiv.org/abs/2002.06563

Rabajante, J. F. (2020). Insights from early mathematical models of 2019-nCoV acute respiratory disease (COVID-19) dynamics. http://arxiv.org/abs/2002.05296

Riddell, S., Goldie, S., Hill, A. et al. The effect of temperature on persistence of SARS-CoV-2 on common surfaces. Virol J 17, 145 (2020). https://doi.org/10.1186/s12985-02001418-7

Schuit, M., Gardner, S., Wood, S., Bower, K., Williams, G. Freeburger, D., \& Dabisch, P. (2020). The Influence of Simulated Sunlight on the Inactivation of Influenza Virus in Aerosols. The Journal of Infectious Diseases, 221(3), 372378. https://doi.org/10.1093/infdis/jiz582

Setti, L., Passarini, F., De Gennaro, G., Barbieri, P., Perrone, M. G., Borelli, M., Palmisani, J., Di Gilio, A., Piscitelli, P., \& Miani, A. (2020). Airborne Transmission Route of COVID19: Why 2 Meters/6 Feet of Inter-Personal Distance Could Not Be Enough. International Journal of Environmental Research and Public Health, 17(8), 2932. https://doi.org/10.3390/ijerph17082932

van Doremalen, N., Bushmaker, T., Morris, D. H., Holbrook, M. G. Gamble, A., Williamson, B. N., Tamin, A., Harcourt, J. L., Thornburg, N. J., Gerber, S. I., Lloyd-Smith, J. O., de Wit, E., \& Munster, V. J. (2020). Aerosol and Surface Stability of SARS-CoV-2 as Compared with SARS-CoV-1. New England Journal of Medicine. https://doi.org/10.1056/nejmc2004973

Yan, J., Grantham, M., Pantelic, J., Bueno de Mesquita, P. J., Albert, B., Liu, F., Ehrman, S., \& Milton, D. K. (2018) Infectious virus in exhaled breath of symptomatic seasonal influenza cases from a college community. Proceedings of the National Academy of Sciences, 115(5), 1081 LP - 1086. https://doi.org/10.1073/pnas.1716561115

Zhang, R., Li, Y., Zhang, A. L., Wang, Y., \& Molina, M. J. (2020). Identifying airborne transmission as the dominant route for the spread of COVID-19. Proceedings of the National Academy of Sciences.

Jones, N. R, Qureshi Z. U., Temple, R.J, Larwood. J.P J, Greenhalgh, T., Bourouiba, L. et al. (2020) Two metres or one: what is the evidence for physical distancing in covid19? BMJ 2020; $370: m 3223$

Appendices. (n.d.). Retrieved October 26, 2020, from https://www.cdc.gov/coronavirus/2019-ncov/php/contacttracing/contact-tracing-plan/appendix.html 